\title{
Révolution, évolution, progrès et régrès chez Élisée Reclus
}

Revolution, Evolution, Progress and Regress in Élisée Reclus's Work

\section{Philippe Pelletier}

\section{(2) OpenEdition}

\section{Journals}

Édition électronique

URL : http://journals.openedition.org/aes/2362

DOI : 10.4000/aes.2362

ISSN : 2258-093X

Éditeur

Laboratoire LISAA

Référence électronique

Philippe Pelletier, "Révolution, évolution, progrès et régrès chez Élisée Reclus », Arts et Savoirs [En ligne], 12 | 2019, mis en ligne le 28 février 2020, consulté le 03 mars 2020. URL : http://

journals.openedition.org/aes/2362 ; DOI : 10.4000/aes.2362

Ce document a été généré automatiquement le 3 mars 2020.

Centre de recherche LISAA (Littératures SAvoirs et Arts) 


\title{
Révolution, évolution, progrès et régrès chez Élisée Reclus
}

\author{
Revolution, Evolution, Progress and Regress in Élisée Reclus's Work
}

\author{
Philippe Pelletier
}

1 La critique du progrès constitue de nos jours une question récurrente, en particulier au sein de l'écologisme - toutes tendances confondues. Elle s'accompagne bien souvent d'une remise en cause des idéologies héritées du XIX siècle, qui sont considérées comme dépassées, voire d'une critique du couple évolution et révolution. S'y adjoint la question de l'insertion du progrès au sein de l'évolution, et de son rapport avec la révolution. Ces questions sont depuis longtemps largement débattues dans la mesure où elles encadrent une lecture du monde, avec ses débouchés idéologiques et politiques porteurs d'enjeux et de rivalités. Avec ses mésinterprétations aussi concernant l'évolutionnisme ${ }^{1}$.

2 Savoir ce qu'en pensait Élisée Reclus (1830-1905) peut être utile. Pas seulement pour des raisons strictement historiographiques, mais aussi pour des raisons analytiques et épistémologiques. À partir du moment où l'on considère en effet que le siècle de la première Révolution industrielle où Reclus a formulé ses idées rencontre notre siècle qui n'est pas post-industriel, mais, au contraire, hyper-industriel, de nouvelles lectures ou relectures sont fertiles. Le recul du grand récit marxiste permet aussi de revenir aux débats fondateurs du socialisme dont notre époque semble redécouvrir les problématiques initiales à travers les nouveaux mouvements sociaux ${ }^{2}$.

\section{Un géographe et un anarchiste}

3 La pensée de Reclus tourne autour des deux topoi que sont la révolution et l'évolution. Cela s'explique par deux raisons. D'une part, en tant que géographe, Reclus évolue d'une géographie physique, à l'origine, vers ce qu'il appellera la " géographie sociale ", en la combinant à la « mésologie $»^{3}$. Entre-temps, il se confronte à ce qu'on peut appeler 
schématiquement la « révolution darwinienne » dont l'épistémologie est centrée sur la théorie de l'évolution.

4 D'autre part, en tant qu'anarchiste, il fait face aux modalités d'action sociale pour lesquelles se pose la question de la révolution. Il y est d'autant plus confronté qu'il fait partie de ce groupe de personnes qui, notamment au sein de la Fédération jurassienne membre de l'Association internationale des travailleurs, contribue à l'élaboration théorique et organique de l'anarchisme. En effet, si, jusque-là, Pierre-Joseph Proudhon (1809-1865) ou Michel Bakounine (1814-1879) parlaient d'anarchie, si Bakounine se disait souvent «socialiste révolutionnaire", en y ajoutant parfois l'adjectif d'« anarchiste », ou de "révolutionnaire anarchiste », la « doctrine anarchiste » ne se formalise véritablement qu'au cours de cette période où Élisée Reclus vit en Confédération helvétique, pendant les années 1870-1880.

5 L'un des temps forts de cette histoire se situe les 9 et 10 octobre 1880 à La Chaux-deFonds. Le congrès de la Fédération jurassienne qui s'y tient se prononce en effet en faveur du "communisme anarchiste", en dépassant le "collectivisme " jusque-là promu par le courant bakouninien. Ce tournant rompt avec l'hostilité des proudhoniens et des bakouninistes pour le communisme synonyme, selon eux, de société monastique.

6 Il est principalement dû à trois hommes qui le théorisent, l'exposent et le font admettre à la Fédération jurassienne : Pierre Kropotkine (1842-1921), Carlo Cafiero (1846-1892) et Élisée Reclus. Il s'explique par plusieurs facteurs - idéologiques, tactiques et stratégiques - notamment au regard des socio-démocrates qui tendent à promouvoir le collectivisme, mais dans un cadre parlementaire, légaliste et étatiste et donc en s'éloignant de l'idéal communiste prôné par le manifeste éponyme ${ }^{4}$. Ce front renversé, paradoxal et presque ironique, où les anciens collectivistes deviennent communistes (mais pas tous...), et où les anciens communistes deviennent collectivistes (mais pas tous...), aura de lourdes conséquences dans l'histoire du mouvement social.

7 Le substantif d'« anarchisme » n'existe pas encore. Ce n'est qu'une vingtaine d'années plus tard qu'il apparaît vraiment et se propage, au-delà de l'adjectif "anarchiste " accolé à un nom. Car, à la fin du XIX siècle, il s'agit de se positionner stratégiquement et de s'identifier idéologiquement vis-à-vis du «syndicalisme révolutionnaire » en plein essor.

8 Cette précision, historiquement nécessaire sous peine d'effectuer un dangereux anachronisme, renvoie à une problématique de fond sur ce que serait, ou ne serait pas, l'anarchie. Selon les uns, celle-ci prend la forme quasi ontologique d'une aspiration humaine éternelle et universelle à la justice et à la liberté en refusant les dominations. Selon les autres, il s'agit d'un horizon qui est certes consubstantiel à la condition humaine, mais qui se transforme en doctrine, soit un ensemble d'analyse du monde, de tactique et de stratégie pour parvenir à l'émancipation individuelle et collective à partir du moment où elle peut être collectivement et internationalement pensée : dans le cadre puis dans le sillage de l'Association internationale des travailleurs.

9 Élisée Reclus balance entre ces deux polarités. Il soutient l'organisation spécifique des anarchistes autour $\mathrm{du}$ " communisme-anarchiste». Utilisant régulièrement le terme d'anarchie dans ses écrits politiques, il n'emploie pas le terme d'anarchisme, comme si l'adjectivation en -isme risquait de faire système, de contraindre la pensée et le mouvement qui s'y réfère. 


\section{Évolution et révolution : deux faces d'un même phénomène}

10 Élisée Reclus est également confronté à la pensée et à la pratique marxistes qui, elles aussi, réfléchissent sur l'idée d'évolution et, surtout, de révolution, tout en gagnant du terrain au sein du mouvement social. Quand il aborde le sujet, notamment dans son seul livre expressément politique, L'Évolution, la révolution et l'idéal anarchique (1898), il ne se réfère toutefois pas directement à Marx ni au marxisme, restant plutôt sur un plan philosophique et stratégique général ${ }^{5}$. Mais l'arrière-plan idéologique et " politique » est clair.

11 À la fin de sa vie, dans le dernier chapitre de son dernier ouvrage, L'Homme et la Terre (1905), Reclus aborde plus précisément la question du progrès, qui sert d'ailleurs d'intitulé au chapitre concerné6. Il s'appuie beaucoup sur les réflexions de son ami en géographie comme en anarchie, Léon Metchnikoff (1838-1888), qui, après un séjour au Japon, s'est installé en Suisse comme son secrétaire pour l'aider à rédiger la Nouvelle Géographie universelle (1880-1888). Metchnikoff écrit notamment deux textes importants sur le progrès ${ }^{7}$.

12 S'y ajoutent les échanges avec un autre ami, également compère en géographie comme en anarchie, Pierre Kropotkine, vivant également en Suisse romande au cours de la même période (1878-1881). Les discussions entre les trois géographes anarchistes vivant au contact des militants de la Fédération jurassienne sont nombreuses, profondes et cruciales.

13 Chez Élisée Reclus, évolution et révolution ne sont que les deux faces d'un même phénomène, dont seul le rythme change. L'évolution, « mouvement infini de tout ce qui existe, transformation incessante de l'Univers et de toutes ses parties depuis les origines », est « synonyme de développement graduel, continu, dans les idées et dans les mœurs »; elle intègre " la révolution qui implique des changements plus ou moins brusques dans les faits $»^{8}$. «La science ne voit aucune opposition entre ces deux mots » 9 .

14 Sa conception de l'évolution s'appuie sur celle de Lamarck et, surtout, de Darwin. Reclus est même l'un des premiers lecteurs du savant britannique en France, ce qui est passé inaperçu. En effet, dans l'un de ses tout premiers articles de géographie, paru en 1865 dans la Revue des Deux Mondes, il cite deux travaux de Darwin, alors pratiquement inconnus en France, ainsi que leur auteur de façon générale ${ }^{10}$.

15 Ces deux écrits ne seront d'ailleurs disponibles en français qu'en 1877 et 1902, respectivement ${ }^{11}$. La question des récifs coralliens, qui forme le sujet du premier article, ne sera débattue qu'une quinzaine d'années plus tard par les géographes français, en particulier par Paul Vidal de la Blache (1845-1918). Cette nouvelle figure de la géographie en France traite alors Darwin de façon étonnante en mentionnant certes son nom, mais, d'après Olivier Soubeyran, sans citer sa théorie ${ }^{12}$.

16 À l'affût de toutes les connaissances et bien informé, Reclus se montre donc d'emblée attentif à Darwin et à ses futurs travaux. Par la suite, il se réfère régulièrement à lui, et de façon positive. Dans L'Homme et la Terre, il considère la théorie darwinienne comme acquise, sans estimer toutefois qu'elle ait tout réglé, c'est-à-dire qu'il la voit comme une base incontournable $d u$ progrès scientifique. Il parle même du "darwinisme». 
Plusieurs passages indiquent qu'il est parfaitement au courant des discussions non seulement scientifiques mais aussi idéologiques qui agitent le monde savant à ce sujet ${ }^{13}$.

Le terme d' " évolution » n'apparaît pas chez Darwin avant la cinquième édition, en 1869, de L'Origine des espèces, dont la première édition remonte à 1859, la sixième et la dernière datant de 1872. Mais il est déjà largement utilisé par Lamarck dont la popularité est grande en France.

L'apparition de la théorie darwininienne constitue, on le sait, un véritable choc dans le monde de la pensée, et au-delà. Elle montre que non seulement le monde vivant évolue, mais qu'il le fait aussi par une logique propre : à la fois interne (sa biologie guidée par la compétition entre espèces et par la « lutte pour l'existence ») et externe (ce que Darwin appelle les "external conditions"). Cette logique conforte le matérialisme philosophique et déstabilise l'idée spirituelle ou métaphysique d'un monde qui aurait été créé et guidé par le divin. Elle mécontente ainsi les courants tant conservateurs que religieux. Inversement, elle séduit et encourage les penseurs socialistes prônant le matérialisme. Proudhon, mourant peu après la publication de L'Origine des espèces, n'a pas l'occasion d'en tirer des enseignements. Eugène Fournière (1857-1914) expliquera certes que l'un de ses derniers ouvrages, La Guerre et la Paix (1861), aurait été influencé par Darwin, mais cette hypothèse est affaiblie par le fait que Proudhon n'y mentionne jamais celuici alors qu'il se réfère à de nombreux autres auteurs ${ }^{14}$.

Karl Marx (1818-1883) découvre avec ferveur les premières recherches de Darwin puisqu'il y voit la confirmation de ses propres théories sur une l'implacabilité de l'évolution historique. Venant de lire L'Origine des espèces en 1860, il envoie une lettre à Friedrich Engels (1820-1895), qui lui avait signalé par courrier l'importance de cet ouvrage, et lui affirme que "c'est dans ce livre que se trouve le fondement historiconaturel de notre conception $»^{15}$.

21 On sait qu'Engels renchérit plus tard sur ce constat, lors de son discours sur la tombe de Marx en 1883, puisqu'il affirme que «de même que Darwin a découvert la loi du développement de la nature organique, de même Marx a découvert la loi du développement de l'histoire humaine ${ }^{16}$. Bakounine applique quant à lui de façon géopolitique le principe darwinien de la « lutte pour l'existence » aux rapports de force entre peuples par le biais des États, ce qu'il craint et dénonce ${ }^{17}$.

Les géographes anarchistes saluent l'apport de Darwin. Pour Élisée Reclus, «toutes les églises catholiques et protestantes ont frémi quand les Lamarck et les Darwin, nouveaux Samson, ont secoué les grands piliers. C'est par les idées mêlées d'actions, non par les prières susurrées au sortir des confessionnaux ou les chapelets égrenés sur le pavé des nefs, que les sociétés se renouvellent » ${ }^{18}$. Selon Léon Metchnikoff, «le brillant mérite de Darwin réside spécialement dans sa surprenante perspicacité avec laquelle son génie a transformé la thèse politico-économique usée dans le principe de régénération, non seulement pour la science biologique actuelle, mais aussi pour la philosophie moderne $»^{19}$. Quant à Kropotkine, « en apportant l'idée de l'évolution dans l'étude de la vie organique, Darwin inaugura une nouvelle ère en philosophie $»^{20}$.

\section{Darwinisme social et social-darwinisme}

Le darwinisme démontrant l'existence d'un mécanisme matériel, naturel, pour l'évolution de la vie végétale et animale, il rend envisageable l'existence d'un 
mécanisme similaire quant à l'espèce humaine. Différents savants matérialistes tentent alors d'en appliquer la logique sur quatre plans : les classes sociales (Enrico Ferri, Paul Lafargue, Thomas H. Huxley, etc.), l'histoire des nations (Walter Bagehot, Claude Royer, etc.), l'histoire des races humaines (Alfred Wallace, Ernst Haeckel, Georges Vacher de Lapouge...) et la géographie (Friedrich Ratzel...).

tellectuels socialistes oscillent dans plusieurs directions à propos du darwinisme. Bien souvent, ils y cherchent une loi de l'histoire. Marx voit celle-ci dans l'évolution des modes de production, tout en critiquant l'esprit de Darwin reflétant les hiérarchies de la classe dominante britannique. Bakounine, à la suite de Proudhon, insiste sur les rivalités de puissance et sur le rôle de la guerre.

Les géographes libertaires (Reclus, Metchnikoff, Kropotkine...) s'avancent en revanche dans une autre direction. Comme la plupart des anarchistes, ils aperçoivent très tôt les dangers que peuvent recouvrir certaines interprétations du darwinisme avec son concept de "lutte pour l'existence». Car deux déductions pernicieuses guettent: consacrer la victoire des plus forts sur les plus faibles (ce qui revient à légitimer le système social existant) ; et nier l'existence de l'entraide parmi les êtres humains (ce qui revient à conforter l'adage hobbesien de l'homme qui serait un loup pour l'homme). Les géographes anarchistes ne s'arrêtent toutefois pas à la critique du seul Darwin. Systématiquement, ils la couplent à celle de Malthus parce qu'ils jugent les deux théories inséparables. De fait, Darwin a reconnu lui-même à plusieurs reprises que sa théorie de la lutte pour l'existence est directement inspirée par Malthus. Après Proudhon, Reclus $(1880,1897,1905)$, Gautier $(1880)$, Metchnikoff $(1886,1889)$ et Kropotkine (1901) dénoncent donc les erreurs scientifiques du pasteur anglais et son arrière-pensée idéologique. Ils y ajoutent également la critique envers Ernst Haeckel (1834-1919), le fondateur de l'écologie qui adopte une vision gladiatrice du darwinisme et qui établit une hiérarchie des races. Généralement, les idées de Darwin ont en effet d'abord transité en France par les écrits de Haeckel, lesquels sont très rapidement traduits en français et bien diffusés (ce sont de véritables best-sellers). Le socialdarwinisme est ainsi souvent confondu avec Haeckel et son écologie.

Parmi les penseurs anarchistes, Émile Gautier (1853-1857) est le premier à publier sur ces questions. Docteur en droit, il s'est lancé dans le journalisme puis rapproché des anarchistes, et il collabore pendant deux ans (1877-1879) avec Jules Vallès (1832-1885) ${ }^{21}$. Il compte parmi les délégués à l'important congrès anarchiste international de Londres (juillet 1881), aux côtés de Kropotkine, Pierre Martin (1856-1916) et Louise Michel (1830-1905). Emprisonné (1883-1885), il renonce à la vie militante après sa libération.

En 1880, il publie Le Darwinisme social ${ }^{22}$. Cet intitulé ne doit pas prêter à confusion. Le «darwinisme social » selon Gautier peut se résumer à l'une de ses formules : «Il faut organiser la lutte contre la lutte pour vivre ». Autrement dit, puisque l'être humain est naturellement capable de lutter pour l'existence, il l'est aussi pour modifier celle-ci vers la justice et l'égalité, puisqu'il s'est mis en "association", avec "un appui réciproque", pour "se protéger et se soutenir mutuellement ${ }^{23}$. Selon lui, le " socialisme - ou la sociologie, ce qui est tout un - ayant pour but unique de corriger les fatalités naturelles » est le moyen pour y parvenir ${ }^{24}$. Il participe ainsi au " progrès » qui «n'a d'autre but que l'expansion de l'individu, c'est-à-dire son développement intégral $»^{25}$. 
Après Gautier, l'expression de «darwinisme social » prendra un sens complètement différent, de concert avec l'inversion de l'épithète et suivant sa formulation ultérieure en anglais de social darwinism ${ }^{26}$. On n'en parle plus dans un sens où le darwinisme participe positivement à l'amélioration de la société, mais, en revanche, dans un sens où la science darwinienne est appelée à justifier les inégalités ou les hiérarchies sociales, ainsi que l'inéluctabilité de l'évolution sociale ${ }^{27}$.

Gautier a rédigé son livre en réaction au cinquantième congrès des naturalistes allemands, auquel participent Haeckel, Nägeli et Virchow ${ }^{28}$. Cette réunion, qui s'est tenue à Munich le 18 septembre 1877, a eu un grand retentissement en raison des affrontements tournant autour de la signification politique du darwinisme.

Rudolph Virchow - médecin, ancien professeur de Ernst Haeckel, fondateur du Parti progressiste allemand (1848), député de Prusse (1862-1902) et du Reichstag (1880-1893) - y affirme ainsi que «le darwinisme mène au socialisme». Dans le cadre du Kulturkampf, notion et politique qu'il a lui-même suggérées aux dirigeants bismarckiens en 1872 , il alerte ceux-ci de la menace que cette nouvelle science apporterait ${ }^{29}$. Selon lui, «la médecine est une science sociale, et la politique n'est rien de plus que la médecine pratiquée en grand ».

Ernst Haeckel et Oscar Schmidt, partisans de l'inégalité des pays dérivant de l'inégalité des races, choisissent un autre angle d'attaque. Ils affirment l'incompatibilité entre l'aspiration égalitaire du socialisme et la loi de l'hérédité ${ }^{30}$. Pour Haeckel, rétorquant que le darwinisme est «foncièrement aristocratique ", "c'est un fait qu'il n'y a aucune doctrine scientifique qui proclame plus ouvertement que la théorie de l'évolution que l'égalité des individus, vers laquelle le socialisme tend, est une impossibilité ; que cette égalité chimérique est une contradiction absolue avec la nécessité et, en fait, l'inégalité universelle des individus $»^{31}$.

Reclus évoquera également le congrès de Munich, mettant dos-à-dos le prétendu progressisme de Virchow et la réplique que lui oppose Haeckel. «Haeckel et, avec lui, tous les disciples de Darwin présents au congrès, prétendirent que la théorie préconisée par lui portait le coup de grâce aux socialistes $\|^{32}$.

Et Reclus de conclure :

Malgré les objurgations de Virchow et d'Haeckel, l'histoire continua son cours, et le socialisme fit son entrée dans le monde parallèlement au darwinisme qui pénétrait dans la science. Les deux révolutions se sont parfaitement accordées, et nombreux sont les savants qui nous expliqué, après coup, pourquoi il devait en être ainsi. Il ressort du moins de l'incertitude de leurs prophéties que les pédants groupés en caste intéressée ne représentent nullement la science, et que celle-ci se développe sans leur concours officiel dans les mille intelligence des hommes qui cherchent isolément, passionnés seulement pour le vrai. ${ }^{33}$

\section{De la « sélection naturelle » à l'« entraide »}

Gautier critique vigoureusement Haeckel après sa dénonciation de Malthus. Il en reproduit in extenso le passage cité précédemment, critiquant tous ces savants qui prétendent constater "que l'égalité des individus est une impossibilité, qu'elle est, cette égalité, chimérique, en contradiction absolue avec l'inégalité nécessaire, et existant partout en fait, des individus $»^{34}$. 

37

Gautier reproche à Haeckel une «politesse dédaigneuse, ne connaissant et ne voulant connaître de l'homme que le côté physiologique » qui a « par voie d'analogie transporté le système entier du darwinisme sur le terrain politico-social ». Autrement dit, il dénonce clairement cette naturalisation du social qui, de nos jours encore, sème la confusion sous d'autres formes. Quant à Reclus, dans une correspondance de 1888, il range Haeckel parmi « la "garde du corps" des Hohenzollern » et parmi ces naturalistes qui » mettent en avant des sophismes pour justifier les inégalités dont ils profitent $»^{35}$.

Gautier ne rejette pas le darwinisme. Il souligne qu'il s'agit d'une hypothèse qui, comme toute science, peut être amendée, mais l'utilisation qui en est faite via le principe de la « sélection naturelle». Car

on comprend aisément le parti qu'ont pu en tirer les champions de l'inégalité sociale. Abandonnant désormais les arguments usés de leurs prédécesseurs, ils substituent aux dogmes indiscutables du péché originel et de la chute de l'homme les dogmes démontrables de la concurrence vitale et de la sélection naturelle. Le livre de Darwin va devenir la Bible des nouveaux exploiteurs : ce sera par et au nom de la science que le sacrifice des faibles s'accomplira! ! $^{36}$

D'un autre côté, Gautier reconnaît que grâce à Darwin on peut affirmer que

tous les phénomènes naturels deviennent d'autant plus facilement et profondément modifiables que leurs lois naturelles ont été mieux connues. [...] Plus l'humanité avance, plus elle s'affranchit des fatalités naturelles. La réaction contre la nature est proportionnelle au progrès, dont elle est en même temps l'indice le plus significatif.

Cette idée de «progrès » qu'avance Gautier dans son interprétation du darwinisme est reprise par les géographes anarchistes.

Peu de temps après, Léon Metchnikoff, qui connaît les recherches de son frère cadet Ilya Metchnikoff (1845-1916), zoologue, biologiste et futur lauréat du Prix Nobel de médecine (1908), renchérit ainsi sur cette approche. Il souligne que «c'est seulement depuis "l'époque darwinienne" et l'examen approfondi de la notion de progrès par les naturalistes que ce mot a acquis un sens précis, indépendant des systèmes métaphysiques et du verbiage d'école $\aleph^{38}$.

2 Lorsqu'il questionne les rapports entre civilisation et progrès, entre individu et société, ainsi que la "philosophie de l'histoire», en évoquant plusieurs auteurs (Mougeolle, Comte, Darwin, Baer, Spencer...), Metchnikoff conclut qu'« au progrès naturel, qui consiste en une sériation des phénomènes naturels où, à chaque étape de l'évolution, la force se manifeste avec une variété et une intensité croissante ", correspond un progrès social, puisque l'être humain fait partie de la nature tout en la dépassant. À la biologie qui s'occupe des espèces vivantes correspond «la sociologie, science qui s'occupe des phénomènes de la vie collective ${ }^{39}$.

Or « la coopération, voilà la caractéristique principale de la vie sociale $»^{40}$. Évoquant les travaux du zoologiste de Saint-Pétersbourg, Karl Fedorovic Kessler (1815-1881) qui considère que l'aide mutuelle est autant une loi de la nature que celle de la lutte pour l'existence, travaux que lira également Kropotkine, Léon Metchnikoff ouvre la voie à une réflexion savante plus approfondie chez les anarchistes sur cette question.

44 Comme on le sait, c'est Pierre Kropotkine qui développera cette idée d'aide mutuelle avec le plus d'insistance, et de concert avec Élisée Reclus. Il le fera notamment à partir de la publication de l'« article atroce ", comme il l'appelle, rédigé en 1888 par Thomas H. Huxley (1825-1895) et intitulé The struggle for existence: a program ${ }^{41}$. 
Dès son article de 1886, Léon Metchnikoff parle d'association et de coopération. Il souligne combien par sa « sociologie » Auguste Comte (1798-1857) prend en compte « ce qu'il appelle les instincts altruistes, supposant que ceux-ci sont inhérents à chaque être vivant ${ }^{42}$. Pour désigner ces phénomènes, Élisée Reclus suggère une dizaine d'années plus tard d'employer le terme d'«entraide» dans un article de 1897 sur La Grande famille, qui est consacré au végétarisme. Il le fait à nouveau en 1898 dans ses Pages de sociologie préhistorique, qui seront reprises dans L'Homme et la Terre ${ }^{43}$. Il le propose à Kropotkine pour traduire l'expression anglaise de mutual aid que celui-ci utilise dans la première édition en anglais de son livre (1902), ensuite publiée en français (1906).

Tandis que Kropotkine et Reclus s'efforcent de montrer l'existence de l'entraide au sein des espèces animales et au sein des sociétés humaines, Metchnikoff insiste sur le progrès qu'elle représente, dans une optique darwinienne, sociale et anarchique. Car «l'association ou la coopération, c'est-à-dire le concours de forces plus ou moins individualises et tendant vers un but commun, apparaît avec les premiers organismes polycellulaires, presque au début même de la série biologique $»^{44}$.

7 Puis, «par la nécessité physiologique résultant de la différenciation » (évoquée par Baer), «le perfectionnement ou le progrès du lien social, débutant dans la série sociologique par la coercition pure et simple, s'achemine vers le caractère de plus en plus psychologique et libre des unions contractées ${ }^{45}$. Parmi ces unions, Metchnikoff mentionne en premier lieu la reproduction sexuelle.

Puis il conclut: "Le progrès sociologique est donc en raison inverse de la coercition déployée, de la contrainte ou de l'autorité, et en raison directe du rôle de la volonté, de la liberté, de l'anarchie. Proudhon l'avait d'ailleurs démontré ${ }^{46}$. Autrement dit, l'être humain évolue de l'empire de la nécessité vers celui de la liberté, vers l'anarchie.

\section{Critique de la philosophie de l'histoire}

ne faudrait cependant pas croire que, pour les trois géographes anarchistes, une telle évolution serait inéluctable, et que leur vision serait purement optimiste. Certes, Kropotkine est, parmi les trois, celui qui se montre le plus confiant, à la fois par une vision mécanique, ce que lui reprocheront d'ailleurs des anarchistes comme Errico Malatesta (1853-1932), et par une croyance en l'imminence de la révolution.

50 Il n'est d'ailleurs pas le seul à l'annoncer à cette époque. Reclus conclut ainsi son livre par « les grands jours s'annoncent. L'évolution s'est faite, la révolution ne saurait tarder $\aleph^{47}$. De fait, l'histoire ne leur donne pas tort puisque, après le prélude de 1905, éclate la révolution russe en 1917, sans parler de la révolution mexicaine, trop souvent oubliée, en 1910.

51 Mais cet optimisme n'est pas synonyme de fatalisme : l'histoire n'a pas de sens, rien n'est en réalité écrit. Metchnikoff et Reclus qui insistent sur ce point introduisent alors une critique radicale de la "philosophie de l'histoire » en tant que vision téléologique. Léon Metchnikoff reprend ainsi l'exclamation du philosophe Francisque Bouillier (1813-1899) - mais cette "philosophie de l'histoire» existe-t-elle donc? - et sa réponse: "J'ai beau chercher, [...] je n'y trouve rien qui soit susceptible de démonstration. ${ }^{48}$

Élisée Reclus propose alors une conception originale de l'histoire, non séparée de la géographie, comme étant une dynamique faite de "progrès » et de "régrès ». Il 
s'inspire explicitement du philosophe napolitain Giambattista Vico (1744-1803) et de sa théorie sur les corsi et les ricorsi, mais en la rénovant ${ }^{49}$.

Vico a été traduit et introduit en France par l'historien Jules Michelet (1798-1874) qui publie en 1827 sa Science nouvelle (Scienza nuova, éd. or. 1725-1744) et, en 1835, des Euvres choisies. Vico a en partie inspiré Proudhon dans son élaboration de la " dialectique sérielle ». Il y occupe « une grande place dans ses extraits », car Proudhon « l'admire et il lui doit certainement beaucoup. Il connait ses œuvres par la traduction de Michelet » dont il a suivi les cours au Collège de France de 1838 à $1841^{50}$. En 1851, il entreprend d'ailleurs une correspondance avec Michelet et noue des liens avec lui.

Élisée Reclus a pris connaissance de Vico grâce à Proudhon, ou grâce à Michelet directement. Il connaît en effet personnellement l'historien qu'il côtoie à un moment de sa vie, en 1863, notamment parce que l'une de ses sœurs, Louise Reclus (1839-1917), s'est remariée avec le gendre de Michelet, Alfred Dumesnil (1821-1894), devenu veuf ${ }^{51}$.

Comme la traduction par Michelet de corsi et ricorsi en progrès et en régrès ne rend pas exactement compte des concepts de Vico, Reclus les évoque aussi en italien ${ }^{52}$. Il ne prend pas la pensée de Vico en bloc et en critique la simplicité.

Ainsi Vico, dans sa Scienza Nuova, nous montre les sociétés évoluant pendant la série des âges par corsi et ricorsi, c'est-à-dire par progrès et régrès réguliers, décrivant des cercles dans le temps et ramenant toujours à un même état de choses après l'achèvement du circuit. C'est là une conception un peu enfantine et nul disciple de Vico n'a pu l'admettre sans la modifier: il est trop évident en effet que l'on ne saurait citer aucune période de l'histoire qui reproduise identiquement une autre période..$^{53}$

Reclus écarte l'idée du retour à un point de départ pour garder le principe d'un progrès jamais définitif, et qui contient toujours des éléments de régrès. Il ajoute plus loin qu'« on parle plus volontiers d'une "spirale de civilisation" dont les cycles, sans cesse agrandis, se développent indéfiniment pendant le cours des âges $»^{54}$. Finalement, «la théorie, jadis fameuse, de Vico sur les corsi et les ricorsi, le flux et le flux des évolutions historiques, se trouve ainsi écartée de la discussion comme l'hypothèse du déplacement successif des centres de culture $"^{55}$. Même l'image de la spirale ne rend pas compte de "l'enchevêtrement infini des faits historiques " ${ }^{56}$.

Reclus applique cette dynamique de la spirale aussi bien à l'évolution des civilisations, notamment dans leur rapport avec le milieu dont la dégradation (dessication, déforestation) peut conduire à leur ruine, qu'à la théorie politique, où l'évolution est inséparable de la révolution. La révolution elle-même n'est pas exempte de brutaux retours en arrière. Cette dernière analyse inspirée de la Révolution française chez Reclus se vérifiera en Russie, en Chine et en moult endroits.

En se défaisant de toute philosophie de l'histoire, Reclus, comme Metchnikoff, introduit immédiatement une dimension spatiale, géographique et mésologique. Il évoque la géographie du temps long en faisant correspondre "l'amélioration générale de l'humanité pendant la période historique » aux « autres cycles de la vie terrestre » qui suivent une autre temporalité et qui ont d'autres effets spatiaux ${ }^{57}$.

Parmi les «temps géologiques», il évoque l'expérience du «refroidissement climatique » ou celle de "la dessication ». Autrement dit, il reconnaît la capacité des êtres humains à être des "agents géologiques" comme il l'écrit ailleurs de façon positive, anticipant ainsi sur la notion actuelle d'anthropocène ${ }^{58}$. 
60 Ainsi, «en se limitant à la seule perspective que présente l'évolution spéciale de l'homme et des animaux qui l'entourent, il est certain que, des origines connues jusqu'à l'époque actuelle, notre monde humain s'est développé de manière à réunir ses groupes épars en une société générale de plus en plus cohérente, et à former avec la Terre qui le porte un tout de plus en plus intime. C'est là ce que, dans leur conception particulière et subjective, les hommes appellent "le progrès" "59.

61 L'unité de la Terre (l'interrelation des écosystèmes dirait-on de nos jours), l'unité de l'humanité (la société mondiale) et leur combinaison « intime », voilà le mot d'ordre de la géographie reclusienne. Et, dans cette "société mondiale ${ }^{60}$, il existe différents niveaux de « développement » et de " progrès » sans que l'on puisse attribuer un degré de supériorité à tel ou tel peuple, ou «nation » puisque jouent également les régrès. Autrement dit, les sociétés dites « civilisées » ou « progressives » ne sont pas celles que l'on croit. Élisée Reclus évoque alors le cas des peuples dits premiers qu'il appelle «nos frères primitifs $»^{61}$.

\section{Nos « frères primitifs »}

L'étude des sociétés dites "primitives » qu'Élisée Reclus et son frère Élie (1827-1904) présentent avec soin et insistance dans leurs travaux, constitue un enjeu au sein de la théorie de l'évolution rapportée à l'être humain, là où l'anthropologie, l'histoire et la géographie se mélangent. Élisée Reclus, qui voit dans les peuples premiers un exemple prototypique de la diversité des hommes et des espaces, y puise sa méfiance envers toute théorie rigide et téléologique de l'histoire.

La vision de l'anthropologie ou plus exactement de l'histoire anthropologique conçue par les frères Reclus se distingue ainsi de celle que formule Friedrich Engels. Celui-ci s'appuie sur les travaux de l'anthropologue américain Henry Lewis Morgan (1818-1881), en particulier dans L'Origine de la famille, de la propriété privée et de l'État $(1884)^{62}$. Élisée Reclus et Léon Metchnikoff refusent également de reprendre la distinction entre Naturvölker et Kulturvölker, contrairement à d'autres géographes de leur temps comme Friedrich Ratzel (1844-1904) ou Vidal de la Blache

64 La dénomination de l'objet d'étude pose aux anarchistes un problème qui existe encore au-delà d'eux : doit-on parler de "peuples primitifs », de "peuples premiers » ou bien d'autre chose? Il n'est pas sûr que les géographes anarchistes du XIX siècle ou les anthropologues anarchistes qui leur sont contemporains (Élie Reclus, Mécislas Goldberg) aient été autant gênés que cela par la question.

Peut-être même peut-on leur appliquer la réflexion contemporaine d'Alain Testart: «On voudrait nous faire accroire que le seul fait de parler d'archaïsme, de primitivité ou d'ancienneté implique une dévalorisation de ce qui est ainsi désigné. Mais pourquoi donc? Faut-il valoriser exclusivement la nouveauté et la modernité ? $»^{63}$ De fait, partant des deux polarités théoriques que sont les "civilisés » et les "primitifs", Reclus considère la société des uns comme faite de "complexité» et d' hétérogénéité ", générant "incohérence » et " contradictions ", et des autres faite de « simplicité » et d' " homogénéité ", générant « cohérence » et « diversité » ${ }^{64}$. Et dans la vie d'Élisée Reclus à en croire tous ceux qui l'ont côtoyé, « simplicité » n'est pas un défaut, c'est une qualité ! 


\section{ainsi l'originalité de l'histoire politique des peuples africains, notamment ceux de} langue bantoue.

Quoi qu'en aient dit plusieurs écrivains, d'après lesquels un des contrastes essentiels entre les Noirs et les autres races de l'Ancien Monde serait l'incapacité des premiers à se grouper en sociétés politiques considérables, les Bantous ont fondé de grands États et quelques populations en offrent un exemple dans le bassin congolais. ${ }^{75}$

Alors que certains hommes politiques français affirment de nos jours que l'Afrique n'est pas encore entrée dans l'histoire, Reclus répond en quelque sorte par anticipation à ces inepties un siècle et demi auparavant.

73 Il ne s'agit toutefois pas pour les anarchistes d'iréniser les sociétés primitives du passé comme du présent. Bakounine pointe ainsi l'anthropophagie, bien que se méprenant sur sa véritable signification. En revanche, tenant des comptes des nouvelles connaissances scientifiques, Kropotkine aborde les pratiques d'infanticide ou de cannibalisme en se demandant s'il n'y a pas, dans les mœurs des sociétés européennes, 
des comportements qui effraieraient les peuples primitifs, comme l'égoïsme. Selon lui, "le sauvage n'est pas un idéal de vertu, mais il n'est pas non plus un idéal de “sauvagerie" »". Quant à Élisée Reclus, il critique le mythe du «bon sauvage » et de la "Nouvelle Cythère " ${ }^{77}$.

Parallèlement à ce refus d'irénisation est posé un refus de la fossilisation. Élisée Reclus insiste, dans sa conclusion de L'Homme et la Terre, que, désormais et sauf rares exceptions, tous les peuples de la terre sont en relation les uns avec les autres, et que, sur le plan de la connaissance scientifique ou du projet sociétaire, il s'agit d'un fait incontournable, et crucial. Kropotkine insiste: "Bien entendu, il n'existe plus de peuples ayant conservé entièrement le mode d'existence de ce temps", celui de "la période post-glaciaire la plus reculée $»^{78}$.

\section{Les peuples « primitifs ", l'histoire et le progrès}

En refusant de parler d'“arriérés " et en mettant régulièrement des guillemets à "primitifs ", les géographes anarchistes interrogent la place attribuée à ces peuples tant dans l'histoire - la course du temps - que dans la géographie - uniformité ou diversité du monde. Se fondant à la fois sur les connaissances et les débats de l'époque, qu'ils peuvent difficilement écarter, ils essaient de dégager une nouvelle voie. Bien entendu, certains de leur propos peuvent apparaître obsolètes, vu les connaissances actuelles, ou insuffisamment étayés.

Ils constatent deux choses: les progrès techniques et scientifiques existent, ils constituent des facteurs cruciaux d'émancipation, tant de la religion que de la machine, donc des prêtres, des patrons et par conséquent des dirigeants ; ils sont propagés par la présence européenne, qu'elle soit imposée (impérialisme, colonialisme) ou librement acceptée, voire recherchée (le cas japonais, par exemple, remarqué par Metchnikoff et Reclus $)^{79}$. Il faut alors souligner plusieurs éléments, pour éviter des mésinterprétations.

Le progrès scientifique et technique n'est pas vu comme une panacée si l'élévation morale ou l'égalité socio-économique ne l'accompagnent pas. En outre, par " européen ", Élisée Reclus entend souvent ce qui est "moderne ", à tel point que les deux mots sont presque interchangeables chez lui. Mais son Europe ne se définit pas par ses limites conventionnelles puisque, à plusieurs reprises, il l'élargit à ce qu'il appelle la Maurétanie, c'est-à-dire à l'Afrique du Nord: "Quoi qu'il en soit des perspectives politiques d'ordre secondaire, la Maurétanie est désormais une nouvelle province de la "plus grande Europe" ${ }^{80}$.

Élisée Reclus n'hésite donc pas évoquer sous ces noms « les pays maurétaniens, c'est-àdire la région qu'on peut appeler l'Europe africaine $»^{81}$. Quand il parle de "monde européen », ce n'est pas l'Europe qui envahit l'Afrique du Nord : c'est l'Afrique du Nord qui retrouve l'Europe par le biais de la Méditerranée. Reclus joue ainsi sur le registre de ce qu'on dénomme actuellement l'invention des continents.

Il pousse même le raisonnement plus loin :

De progrès en progrès, la civilisation européenne en est arrivée à la négation de son point de départ. Elle visait à la domination, à la prépondérance, et par ses conquêtes mêmes elle constitue l'égalité. Le monde entier s'européanise : on peut même dire qu'il est déjà européanisé. ${ }^{82}$ 

pratiquement synonyme de " modernité, et « européen » de « moderne ». Même si l'on peut trouver cette extension géographique abusive, elle retrace la course géographique de l'histoire en conclusion cosmopolite et universelle puisque, dans de nombreux passages, Reclus souligne combien les peuples hors de l'Europe ont hâte de se doter des outils modernes.

81 Y voir un européocentrisme classique serait donc une erreur. Le monde européen n'est pas considéré par Reclus comme supérieur en soi, mais comme prépondérant par la dynamique - bonne ou mauvaise - qu'il impulse. Ce qui est d'ailleurs un fait. Reclus garde le qualificatif européen car, en tant que géohistorien, il tient à marquer les origines du phénomène et les caractères spatiaux de sa diffusion.

82 Assurément, la terminologie peut être mal comprise. C'est pourquoi il recourt systématiquement aux termes d'« universel » et d'«humanité ». Il est conscient que, désormais, le phénomène dépasse l'Europe. Il affirme ainsi que "l'équilibre, aujourd'hui, n'est plus européen. Il n'y a plus d'équilibre européen. Il ne peut y avoir qu'un équilibre "mondial" reposant, non sur la puissance d'un seul ou sur la jalousie réciproque des gouvernements, mais sur le respect mutuel des peuples et des individus les uns vis-à-vis des autres, aussi bien que sur la pratique commune de la justice $»^{83}$. Ce propos, soulignons-le, est tenu dans un pays colonisé, à Alger en 1885 : c'est même la conclusion de sa conférence. Reclus reste en outre lucide sur ce qu'il nomme « l'impérialisme à la romaine » dans le dernier volume de L'Homme et la Terre ${ }^{84}$.

83 Se pose donc, pour les géographes anarchistes, la question de la place des "primitifs » à deux niveaux dans ce monde-là : dans la science et dans l'humanité. Rappelons que pour ces savants héritiers des Lumières et du positivisme qu'ils cherchent à dépasser, l'objectif est, pour reprendre la formule de Fernand Pelloutier (1867-1901), fondateur de la Fédération des Bourses du travail, de posséder "la science de son malheur, connaître les causes de sa servitude ». Connaître les "primitifs", c'est comprendre l'humanité dans son ensemble, donc se connaître soi-même.

Comme le souligne Élisée Reclus dans la conclusion de son œuvre, "quand nous comparons notre société mondiale, si puissante, aux petits groupes imperceptibles des primitifs qui ont réussi à se maintenir en dehors des "civilisateurs" - trop souvent destructeurs - nous pouvons être portés à croire que ces primitifs nous étaient supérieurs et que nous avons rétrogradé sur le chemin des âges $»^{85}$. La civilisation de « nos sociétés modernes » peut donc être régressive.

Inversement, elle comporte aussi des éléments relevant de "peuples primitifs » ou de «peuples sauvages». Il pose ainsi la question: "Quelle nation peut se dire complètement dégagée de la sauvagerie première? ${ }^{86}$ Non seulement l'idée de «sauvagerie» ou de «civilisation" est relative, mais ce qu'on entend par "sauvagerie", cette fois de façon positive, existe aussi chez les sociétés dites « civilisées ». Combien d'autres que lui écrivent cela à son époque?

Son frère, Élie Reclus abonde dans le même sens: "Aussi n'hésitons-nous pas qu'en nombre de tribus, dites sauvages, l'individu moyen n'est inférieur, ni moralement, ni intellectuellement, à l'individu moyen dans nos États civilisés. $»^{87} C^{\prime}$ est la conclusion qui vient après une métaphore risquée sur l'intelligence des primitifs qui serait comparables à celle des enfants, que l'on peut mésinterpréter si l'on n'admet pas que les adultes ne sont pas forcément ni « adultes » ni civilisés. 


\section{Progrès, régrès, milieux, civilisation} qu'à sociale ». dans les Andes.
Selon Reclus, le progrès est une amélioration de la condition humaine : il « consiste à trouver l'ensemble des intérêts et des volontés commun à tous les peuples, il se confond avec la solidarité $»^{88}$.

Et dans «tous les peuples », il inclut les «primitifs ». Car «l'homme moderne » peut, grâce à sa « capacité plus grande ", « reconquérir le passé du sauvage dans son milieu naturel antique et l'associer, le fondre harmonieusement avec ses idées plus affinées »,

la condition pour l'homme nouveau d'embrasser tous les autres hommes, ses frères, dans un même sentiment d'unité avec l'ensemble des choses. Voici donc la question sociale qui se pose de nouveau et dans toute son ampleur. Il est impossible d'aimer pleinement le sauvage primitif, dans son milieu naturel d'arbres et de ruisseaux, si l'on n'aime pas en même temps les hommes de la cité plus ou moins artificielle du monde contemporain. ${ }^{89}$

Ce passage résume bien la pensée reclusienne. Le géographe anarchiste admet la modernité, mais celle-ci ne suffit pas à "l'homme nouveau » (expression dont le xx siècle fasciste ou stalinien montrera néanmoins toute la dangerosité). Refusant d'opposer le civilisé au primitif ou de tomber dans la misanthropie, il exprime clairement le fait que la question primitive fait partie intégrante de la "question

Pour Reclus, nulle situation n'est figée puisque chaque milieu étant localement divers, chaque peuplade peut effectuer des choix technologiques et culturels différents malgré des conditions parfois proches. Il récuse donc les théories dites évolutionnistes, alors à la mode, qui, depuis Condorcet, au moins, avec Comte puis avec Spencer, établissent une succession caractérisée et quasi obligée des différents types de civilisation.

1 Il critique «le public qui se laisse aller à répéter comme des vérités acquises des hypothèses commodes et plausibles qui dispensent de réfléchir », telles que les « dix périodes » distinguées par Condorcet dans l'histoire de l'humanité, et qui font d'ailleurs penser aux six stades évoqués par Fourier ${ }^{90}$. Fidèle à son approche selon laquelle l'analyse de la nature n'est pas séparable de celle de l'humanité qui l'habite, et réciproquement, Élisée Reclus rappelle que « l'étude détaillée de la Terre nous prouve que cette succession prétendue des états est une pure conception de l'esprit en désaccord avec les faits $»^{91}$.

Il expose alors une intuition qui ne sera entérinée que plusieurs décennies plus tard grâce aux acquis de l'ethnographie, à savoir que, si la chasse et la cueillette constituent le premier stade de civilisation «sinon universel, du moins normal », l'agriculture a plutôt précédé l'élevage qu'il ne l'a suivi : «L'agriculture n'eut pas besoin pour naître de succéder à l'état pastoral. ${ }^{92}$ Il en donne quelques exemples, dont celui des Incas

Élisée Reclus s'appuie aussi sur l'opinion commune du progrès en se référant d'emblée, dans son chapitre de L'Homme et la Terre, à un historien britannique plutôt classique, Edward Gibbon (1737-1794). Il le cite : «Depuis le commencement du monde, chaque siècle a augmenté et augmente encore la richesse réelle, le bonheur, la science, et peutêtre la vertu de l'espèce humaine. $»^{93}$ 


\section{Il ajoute :}

L'opinion moyenne relative au progrès coïncide bien avec celle de Gibbon [...] : l'amélioration de l'être physique au point de vue de la santé, l'enrichissement matériel et l'accroissement des connaissances, enfin le perfectionnement du caractère, devenu certainement moins cruel, plus respectueux de l'individu, et peut-être plus noble, plus généreux, plus dévoué. ${ }^{94}$

Reclus fait intervenir alors la notion de «civilisation» en parallèle à celle de " progrès ». Comme pour le second terme, qui « pris dans un sens absolu n'a point de signification puisque le monde est infini », le premier peut revêtir, selon lui, plusieurs sens possibles: "Le mot de "civilisation", qu'on emploie d'ordinaire pour indiquer l'état progressif de telle ou telle nation est, comme le terme de "progrès", une de ces expressions vagues dont les divers sens se confondent $»^{95}$. Reclus rappelle donc son sens commun - « raffinement des mœurs ", mais aussi « améliorations matérielles dues à la science, à l'industrie moderne ».

Pour parler de civilisation et partir du sens commun, Metchnikoff avait lui aussi, une quinzaine d'années auparavant, fait appel aux propos d'un autre intellectuel, l'historien Paul Mougeolle qui mobilise la mésologie, comme le fera plus tard Élisée Reclus, pour faire une histoire des peuples et non des grands hommes. Selon Mougeolle, l'expression "complexe" de civilisation "embrasse la totalité des découverts faites et des inventions réalisées ", " la mesure des idées en cours et des procédés en usage ", «le degré de perfection de la science, de l'art et de l'industrie ", "l'état de la famille, de la société et de toutes les institutions existantes $»^{96}$.

Metchnikoff précise toutefois: "Si le perfectionnement technique est incontestablement un des éléments principaux du progrès, il n'est point tout le progrès ». Interfèrent en effet le "véritable progrès dans l'histoire ", c'est-à-dire « les transformations successives du lien social, les variations consécutives des rapports d'homme à homme ».

Reclus ajoute un élément important sur lequel il attire l'attention. Selon lui, « la société actuelle contient en elle toutes les sociétés antérieures à l'état de survivances ", notamment sous forme de "classes superposées" (on se rappellera que Reclus distingue trois lois en géographie sociale: «la "lutte des classes", la recherche de l'équilibre et la décision souveraine de l'individu»). Et ces classes peuvent présenter des « situations extrêmes » avec « un écart saisissant " ${ }^{97}$.

\section{La double critique reclusienne de la religion et du " décadentisme »}

99 Élisée Reclus ne s'illusionne pas sur les vertus du progrès matériel et technique. Ainsi il ironise :

De quels chants de triomphe en l'honneur du progrès n'ont pas été accompagnées les inaugurations de toutes les usines industrielles avec leurs annexes de cabarets et d'hôpitaux! Certes, l'industrie amena de réels progrès dans son cortège, mais avec quel scrupule il importe de critiquer les détails de cette grande évolution $!^{98}$

100 Pour autant, l'enchaînement même de ces deux phrases montre bien qu'il ne récuse pas le progrès en soi. Il va même plus loin en déplorant que "des hommes de haute intelligence nient absolument le progrès " ${ }^{99}$. Ainsi "quelques grands esprits ne se 
contentent point d'admettre ces restrictions capitales à la notion du progrès, ils nient même qu'il puisse y avoir amélioration réelle dans l'état général de l'humanité $»^{100}$.

Reclus dénonce alors les contempteurs contemporains du progrès. Il les subdivise en deux groupes : le premier qu'il nomme - celle des religieux et des religions - et le second pour lequel il ne donne pas de noms précis, mais que l'on peut identifier comme étant le courant des « décadents».

Dans sa critique de la religion, Reclus vise le principe créationniste qui postule une "perfection suprême ", celle du créateur divin. Or celle-ci et celui-ci n'existant pas, le discours religieux ne peut que stigmatiser «la chute ", "la décadence » ou le "régrès fatal ", tout en l'attribuant au péché. Il accompagne de surcrô̂t les intérêts de la classe dominante qui présuppose que "toute révolution, tout changement doit être une chute, un retour vers la barbarie», ce qui conduit au conservatisme, sinon à l'immobilisme ${ }^{101}$.

103 Reclus cite l'historien saxon, luthérien, piétiste et conservateur, Leopold von Ranke (1795-1886), pour qui le progrès coupe «les hommes, assurés d'une amélioration de siècle en siècle" de la "dépendance directe de la divinité » ${ }^{102}$. Il lui oppose le philosophe libertaire Jean-Marie Guyau (1854-1888) pour qui « l'idée du progrès est en antagonisme avec l'idée religieuse $»^{103}$.

104 La critique reclusienne revêt ainsi une double actualité. Elle renvoie à la position de l'Église catholique qui se déclare officiellement hostile au progrès dès l'encyclique Rerum Novarum de Léon XIII (1891), reprise plus tard par Paul VI notamment dans son encyclique Humanae vitae (1968). Elle s'applique également aux tendances religieuses et anti-progressistes de l'écologisme, que l'on trouve par exemple chez Jean-Marie Pelt ${ }^{104}$.

Parallèlement aux religieux, Élisée Reclus distingue d'autres contempteurs du progrès. Selon lui, il existe des hommes effrayés par les bouleversements de la Révolution industrielle qui se mettent à "discourir en prose et en vers sur la "corruption du siècle" » et qui, à propos de l'humanité, "parlent volontiers de sa décadence ». En utilisant ces expressions à la mode, Reclus vise le courant souvent anti-moderniste, parfois lié au symbolisme, qui dénonce la supposée décadence de l'époque et qui se rapproche parfois de l'anarchisme.

Celui-ci voit alors s'entrecroiser le courant individualiste en plein essor et le courant syndicaliste révolutionnaire qui se structure - dont les « décadentistes » ne tardent pas à se détacher vu son caractère jugé trop plébéien ou prosaïque ${ }^{105}$. Parmi eux, citons Anatole Baju (1861-1903), Paul Adam (1862-1920), Adolphe Retté (1863-1930) ou Léo Taxil (1854-1907).

Dès 1885, Le Révolté, journal fondé à Genève par Kropotkine en 1879 puis repris à Paris par Jean Grave (1854-1939) à partir de 1883, avec l'appui financier, moral, politique et épistolaire d'Élisée Reclus, rend compte avec sympathie au début, de ce mouvement du "symbolisme décadent". Mais il déplore déjà le "pessimisme" de ces «jeunes réactionnaires $»^{106}$. Tout en appréciant le talent de certains de ces littérateurs, il reste sceptique quant à leur intérêt pour la question sociale et à la profondeur de leur engagement. Ce que la suite se chargera de démontrer puisque la plupart des décadentistes rompront avec l'anarchisme.

L'enthousiasme initial qu'Élisée Reclus exprime en 1892 pour les Entretiens politiques et littéraires (1890-1893), qui ont été fondés par Adam, Vielé-Griffin et Régnier, et qui 
relaient les décadents, s'est donc singulièrement refroidi en 1905 dans L'Homme et la Terre $^{107}$.

\section{La question du « retour à la nature »}

Élisée Reclus conclut son approche du progrès et de la civilisation en reposant la question de la nature. Une cinquantaine d'années auparavant, il avait déjà constaté qu' 'il se manifeste depuis quelque temps une véritable ferveur dans les sentiments d'amour qui rattachent les hommes d'art et de science à la nature $»^{108}$. Reprenant cette thématique, il part de l'expression de "retour à la nature ", et du mouvement social qui l'accompagne, pour l'élargir à d'autres considérations ${ }^{109}$.

À première vue selon lui, il semble y avoir une contradiction entre l'élaboration des nouveaux "droits de l'Homme et du Républicain», consacrés par la Révolution française, et la recherche, par cette même société moderne, d'un retour « vers les âges heureux et purs des tribus préhistoriques $»^{110}$. De fait, alors que son texte à propos $D u$ sentiment de la nature dans les sociétés modernes de 1866 mettait l'accent sur la découverte des montagnes (ascensions, randonnées, description des paysages...), puis sur les nouveaux rapports entre la ville et la campagne, la conclusion de L'Homme et la Terre envisage d'emblée la question du « retour à la nature » dans son rapport entre lesdits civilisés et lesdits primitifs.

111 Cette convergence permet d'abord d'affirmer l'unité du genre humain, et par conséquent la communauté de ses intérêts ou la possibilité d'un progrès commun. Pour cela, Reclus fait allusion aux "hommes de désir» évoqués par «le "philosophe inconnu" du XVIII ${ }^{\mathrm{e}}$ siècle", alias Louis-Claude de Saint-Martin (1743-1803) dont la pensée illuministe et christologique donne finalement aux êtres humains le pouvoir de dépasser la création divine. Il insiste, dans une veine toute bakouninienne qui sera reprise par l'anarchisme malatestien, sur l'importance de la volonté.

Toutes les nations, y compris celles qui se disent ennemies, ne constituent, en dépit de leurs chefs et malgré les survivances de haines, qu'une seule nation dont tous les progrès locaux réagissent sur l'ensemble et constituent un progrès général. Ceux que le "philosophe inconnu » du XVIII ${ }^{\mathrm{e}}$ siècle appelait les « hommes de désir », c'està-dire ceux qui veulent le bien et qui travaillent à le réaliser, sont assez nombreux déjà, assez actifs et assez harmonieusement groupés en une nation morale pour que leur œuvre de progrès l'emporte sur les éléments de régrès et de dissociation que produisent les haines survivantes. ${ }^{111}$

112 Déclinant sa célèbre épigraphe postulant que «l'homme est la nature prenant conscience d'elle-même", Reclus insiste ensuite sur le fait que le double processus de volonté et de progrès n'est pas naturel, au sens fort du terme.

Le progrès conscient n'est pas un fonctionnement normal de la société, un acte de croissance analogue à celui de la plante ou de l'animal ; il n'éclot pas comme une fleur, mais se comprend comme un acte collectif de la volonté sociale, qui arrive à la conscience des intérêts solidaires de l'humanité et les satisfait à mesure et avec méthode, se consolidant d'autant plus que cette volonté s'entoure d'acquisitions nouvelles. ${ }^{112}$

Après une réfutation des théories de Hegel ou de Brück qui déterminent la course de l'histoire selon un trajet allant de l'Orient à l'Occident, Élisée Reclus pose alors son raisonnement géographique majeur.

114 En effet, avec le progrès, 
l'élément espace a perdu de son importance, car l'homme peut s'instruire et s'instruit en effet de tous les phénomènes du sol, du climat, de l'histoire, de la société qui distinguent les différents pays. Or, se comprendre, c'est déjà s'associer, se confondre en une certaine mesure. Certainement, le contraste existe toujours entre terre et terre, nation et nation, mais il s'atténue et tend graduellement à se neutraliser dans la compréhension des gens avertis. [...] Cette extension même du champ d'études, croissant avec les révolutions et les siècles, constitue un des principaux éléments du progrès : l'humanité consciente s'est constamment accrue en proportion même de l'assimilation géographique des terres lointaines au monde déjà scruté scientifiquement. ${ }^{113}$

Commençant par une allusion fine à la célèbre formulation cartésienne, Élisée Reclus ajoute :

Maîtres désormais de l'espace et du temps, les hommes voient donc s'ouvrir devant eux un champ indéfini d'acquisitions et de progrès, mais, embarrassés encore par les conditions illogiques et contradictoires de leur milieu, ils ne sont point en mesure de procéder avec science à l'œuvre harmonique de l'amélioration pour tous. 114

Autrement dit, il faut science et conscience pour progresser. Mais pour quoi faire?

\section{La « conquête du pain »}

117 L'objectif est « la conquête du pain ». C'est le titre que Reclus a donné en 1892 à l'un des plus fameux livres de Kropotkine. Il en parle déjà lors de sa conférence Évolution et révolution de 1880, qui lui permet d'engager une critique radicale de Malthus. Il y estime en effet que «la terre est dès maintenant riche et plus que riche pour subvenir abondamment à tous les besoins de l'humanité »115. Dans son livre de 1891 qui reprend et développe sa conférence, il reprend l'argument : « La Terre est assez vaste pour nous porter tous sur son sein, elle est assez riche pour nous faire vivre dans l'aisance $»^{116}$.

118 Ce pain n'est pas seulement matériel, il est aussi moral et spirituel. Sa conquête n'est pas passive, mais active. Car « la conquête du pain, telle que le vrai progrès l'exige, doit être réellement une conquête ${ }^{117}$. "L'idéal évolutionniste est le but révolutionnaire ", comme l'indique le sous-titre du chapitre cinq du livre de 1891, et il est porté par les «évolutionnistes conscients ». La finalité est l'éradication de la misère matérielle et morale, son moyen est la solidarité et la libre association - éléments fondamentaux du progrès.

119 Autrement dit,

dans son essence, le progrès humain consiste à trouver l'ensemble des intérêts et des volontés commun à tous les peuples; il se confond avec la solidarité. Tout d'abord, il doit viser à l'économie, bien différent en cela de la nature primitive, qui prodigue les semences de vie avec si étonnante abondance. [...]. Le fléau de la misère est un de ceux que la méthode scientifique, dans la répartition des biens de la terre, serait en mesure de corriger rapidement, puisque les ressources nécessaires à tous les hommes sont en surabondance. ${ }^{118}$

La méthode scientifique, c'est-à-dire celle de la géographie, notamment, voit ainsi tracer sa feuille de route.

Après Descartes, Reclus poursuit ses références aux Lumières en évoquant Condorcet.

C'est aux conquérants du pain, c'est-à-dire aux hommes de labeur, associés, libres, dégagés du patronage, que se trouve remise la cause du progrès. C'est à eux qu'il reviendra d'introduire enfin la méthode scientifique dans l'application aux intérêts 
sociaux de toutes les découvertes particulières, et de réaliser le dire de Condorcet, que «la nature n'a mis aucun terme à nos espérances ». Car, ainsi que l'a dit un autre historien sociologue [Taine], « plus on demande à la nature humaine, plus elle donne; ses facultés s'exaltent à l'œuvre, et l'on n'aperçoit plus de limites à sa puissance ». ${ }^{119}$

Élisée Reclus livre ses derniers mots.

Ce n'est pas tel ou tel stade de l'existence personnelle et collective qui constitue le bonheur, c'est la conscience de marcher vers un but déterminé, que l'on veut et que l'on crée partiellement par sa volonté. Aménager les continents, les mers et l'atmosphère qui nous entoure, «cultiver notre jardin " terrestre, distribuer à nouveau et régler les ambiances pour favoriser chaque vie individuelle de plante, d'animal ou d'homme, prendre définitivement conscience de notre humanité solidaire, faisant corps avec la planète elle-même, embrasser du regard nos origines, notre présent, notre but rapproché, notre idéal lointain, c'est en cela que consiste le progrès. Oui nous avons progressé depuis le jour où nos ancêtres sortirent des cavernes maternelles, pendant les quelques milliers d'années que constitue la courte période consciente de notre vie. ${ }^{120}$

La dynamique reclusienne de «milieu-espace » et de "milieu-temps », pour familière qu'elle puisse paraittre à des habitués de l'histoire braudélienne ou de la géographie contemporaine, est, de ce point de vue, fondamentale, et riche de possibilités. Comme Élisée Reclus l'écrit, la géographie n'est pas donnée une fois pour toutes, elle est mobile, évolutive, mouvante. Cette dialectique renvoie à nouveau à la question du déterminisme, à propos duquel l'anarchisme se distingue du marxisme et du libéralisme.

Car, dans le sillage d'un hégélianisme surdimensionné, le marxisme accorde en revanche une prépondérance à l'histoire, qu'il conçoit comme un parcours linéaire, avec un début et une fin programmée, avec des étapes obligées. Il en fait une métaphore quasi sociobiologiste de la vie et de la mort d'un individu, en y ajoutant une dimension téléologique pratiquement inspirée des religions du livre reformatées en scientisme.

L'anarchisme ne s'appuie pas sur une philosophie de l'histoire proclamant soit l'arrivée du capitalisme à son stade final, soit l'imminence d'une catastrophe écologique planétaire. En effet, comme le remarque Nicholas Spencer, «la croyance dans l'histoire garantit l'autorité politique, car le changement dans le temps implique le besoin d'un corps centralisé pour guider le processus de changement $»^{121}$. À la conception linéaire de l'histoire, s'oppose, à partir de Vico, Proudhon et Reclus, le principe de progrès et de régrès. Autrement dit, d'avancée et de recul social, notamment en termes de civilisation.

Les termes de civilisation et de progrès n'effraient donc pas Reclus pour qui la civilisation est un niveau d'avancement matériel et spirituel de l'humanité à condition que celui-ci profite à tous. Les contempteurs contemporains du progrès, qui confondent innovation technologique et système capitaliste, sont en réalité prisonniers de la conception même des libéraux et des marxistes qui, tous les deux, à leur façon, postulent un sens de l'histoire. 


\section{Cultiver notre jardin}

127 Même si la théorie de l'évolution trace un cadre globalement progressiste, l'histoire n'est pas programmée comme le démontre la géographie des peuples par leur variété et leurs différentes adaptations au milieu. De fait, les progressistes et les civilisés ne sont pas forcément ceux que l'on croit : les sociétés modernes ont beaucoup à apprendre de « nos frères primitifs » - et réciproquement. La pensée d'Élisée Reclus ne relève donc pas d'un évolutionnisme tel qu'on l'entend classiquement si l'on reprend la catégorisation - schématique mais pratique - proposée par Salvador Juan (ethnocentrisme, progressisme, productivisme, scientisme) qui redéfinit son contenu de façon parfois erronée ou excessive $e^{122}$.

Ethnocentrique, Élisée Reclus ne l'est pas puisqu'il valorise l'existence et l'apport des peuples " primitifs ", qu'ils considèrent comme des « frères ", donc à égalité, y compris sur le plan politique. Comme nous l'avons vu, son utilisation régulière de l'adjectif « européen » ne doit pas nous induire en erreur puisque les limites géographiques qu'il attribue à l'Europe vont au-delà des limites conventionnelles. Même un pays comme le Japon finit par y rentrer, par faire partie du «monde européen ». Le Japon le fait de surcroît après une "révolution", ainsi que l'appelle Reclus à la suite de Metchnikoff qui définissent ainsi l'instauration du régime de Meiji en 1898.

Reclus utilise en outre le terme d'« européen» dans le sens de «moderne » comme nous l'avons vu. Pourquoi insiste-t-il davantage sur l'un et moins sur l'autre? Il ne l'explique pas, mais on peut déduire de ses propos qu'il cherche à déconstruire les catégories géographiques car celles-ci risquent, en étant figées, de forger de fausses réalités, de créer des barrières entre les peuples. En outre, les termes de "moderne " ou de "modernité » ne conviennent plus à partir du moment où l'on raisonne en termes de progrès et de régrés, sinon par simple commodité. Il faut donc jongler avec le vocabulaire, quitte à être ambigu parfois.

Du coup, Reclus ne peut guère être classifié de «progressiste » dans un sens classique et étroit, même si, de fait, il aspire pour lui, pour l'humanité et les êtres vivants à un monde meilleur, refusant d'attendre le paradis dans l'au-delà. Il ne s'agit pas du " progressisme " marxien cherchant à postuler scientifiquement que l'histoire nous amène fatalement vers le socialisme ou le communisme, ni même du progressisme libéral reposant principalement sur la technique. Mais, comme Reclus croit à la possibilité et même à l'imminence d'une révolution sociale, sa pensée anarchiste y converge logiquement.

131 Le monde étant désormais connu et en interaction, le projet émancipateur selon Reclus passe par la prise en compte de ses interrelations fondées sur un effort non pas de pouvoir mais de "puissance» (c'est le mot qu'il utilise - de «capacité » dirait Proudhon) et de « volonté ».

132 Par " productivisme ", Juan Salvador entend le fait de " produire pour produire ", mais, comme bien d'autres personnes qui reprennent ce concept forgé par les personnalistes français du groupe Ordre nouveau des années 1930 (Dupuis, Marc, Rougemont, DanielRops...), il s'agit d'un contresens. En effet, le système économique dominant, le capitalisme, produit pour vendre, pour dégager de la plus-value et du profit, quitte à, parfois, vendre à perte, donner ou détruire pour rétablir le marché123. 
133 En revanche, en se positionnant radicalement contre le malthusianisme, de concert avec Metchnikoff et Kropotkine, Élisée Reclus plaide clairement pour une augmentation de la production afin de satisfaire les besoins de toute l'humanité. Il l'estime physiquement possible, d'autant que la répartition des richesses actuelles n'y suffira pas. Ce constat le distingue fondamentalement de tous les courants écologistes actuels qui sont peu ou prou malthusiens, quoiqu'ils en disent.

De ce point de vue, on a du mal à comprendre pourquoi certains auteurs s'évertuent à faire de Reclus un "écologiste» avant l'heure, à moins de s'empêtrer dans l'anachronisme ou la récupération politico-idéologique. En outre, sur de nombreux autres points (le progrès, la technique, la critique de l'État et du parlementarisme), Reclus n'a guère à voir avec l'actuel courant « vert ».

Enfin, le «scientisme » semble qualifier la pensée reclusienne. Il est sûr que Reclus, ainsi que Metchnikoff, Kropotkine ou tant d'autres anarchistes de son temps, mise sur la science, notamment « la science de notre malheur ", comme nous l'avons souligné, pour émanciper l'individuel et le social, ne serait-ce que parce qu'elle permet de sortir de l'obscurantisme religieux et de la domination des prêtres, de tous les prêtres. Certes, nombre de ses propositions en matière d'aménagement de l'espace s'apparentent à du saint-simonisme (création de routes, de canaux, de chemins de fer,etc.).

136 Mais Reclus ne considère pas la science comme un fétiche, ni comme un savoir définitivement constitué. En affirmant que « la science n'est pas : elle se fait, le savant $\mathrm{du}$ jour n'est que l'ignorant du lendemain ${ }^{124}$, Reclus touche plusieurs points sensibles. Il ne parle pas de science désincarnée, mais de savants, c'est-à-dire de personnes socialement situées qui font de la science, qui savent un jour mais pas toujours.

137 Car la science évolue, ce n'est pas un dogme. Les savants doivent donc rester humbles, ce qu'ils n'aiment pas et ce qui contrevient à leur position sociale souvent dominante, arrogante et dirigeante : ce que dénonçait précisément Bakounine quand il critiquait le "gouvernement des savants ». Reclus fait même un distinguo entre « la science vécue et la science officielle $»^{125}$.

138 Il serait trop facile de reprocher à Reclus, comme à d'autres penseurs, de ne pas avoir suffisamment critiqué ceci ou cela, d'avoir épousé une partie de l'air de son temps. Le géographe anarchiste n'a certes pas approfondi certaines des pistes qu'il a empruntées, notamment à propos de la mésologie ${ }^{126}$. Ses tâches étaient parfois ailleurs (la construction d'un globe terrestre, par exemple) ainsi que les priorités intellectuelles (combattre le mysticisme et le social-darwinisme, le déterminisme géographique, la géopolitique née du sol, etc.), tandis que le contexte général, hostile à la pensée libre, favorable au social-darwinisme ou à la philosophie de l'histoire, fermait davantage les portes qu'elle n'en ouvrait.

139 Le qualifier négativement d'« évolutionniste » comme le fait hâtivement Salvador Juan relève en fait d'un enjeu récurrent qu'a relevé Alain Testart. En effet, déjà, à la fin du $\mathrm{XIX}^{\mathrm{e}}$ siècle, l'évolutionnisme est mal vu. En ne se bornant pas à opposer des états antérieurs à des états postérieurs mais en « établissant toujours une certaine continuité entre l'antérieur et l'ultérieur », il rapproche "les deux états, d'une façon dangereuse pour l'état final qui se voudrait non seulement supérieur mais bien d'essence radicalement distincte de l'autre. [...] L'évolutionnisme est porteur d'une menace pour les valeurs traditionnelles de notre civilisation, et on peut se demander si ce n'est pas précisément à ce titre qu'il a été combattu » ${ }^{127}$. En croisant " primitif » et «civilisé » 
sans leur faire correspondre "progrès " et "régrès", Élisée Reclus se retrouve précisément dans cette situation où il est critiqué. Il tombe sous le coup des modernistes comme des décadentistes, des marxistes comme des libéraux.

Le « retour à la nature » constaté à son époque par Reclus, qui fait ainsi écho à la nôtre, n'est pas contradictoire avec une autre organisation et gestion des milieux. «Cultiver notre jardin terrestre » va de pair avec la « conquête du pain » matériel et moral. Le projet libertaire reclusien doit donc tout autant se méfier de ceux qui ruinent "l'harmonie secrète établie entre la terre et les peuples qu'elle nourrit» que des » dénigreurs» du progrès, des «discoureurs, des esprits chagrins» ou des "prophètes de malheur", souvent des religieux ou des décadentistes qui annoncent la fin du monde pour mieux exercer leur pouvoir.

141 Admettre le régrès, ce n'est pas non plus tomber dans le discours rétrograde qui, depuis les opposants de la Révolution française, De Bonald, Malthus, Chateaubriand, aux théoriciens de la décadence comme Oswald Spengler (1880-1936), avec son Déclin de l'Occident (1918-1922) et sa théorie de l'« historicisme», ou comme Ludwig Klages (1872-1956), cultivent en fait une nostalgie suspecte.

On reconnaîtra sans peine dans ce dernier constat la dérive actuelle des catastrophistes et de ceux qui pavent la voie à un "capitalisme vert ». Car loin de s'effondrer, le capitalisme se recompose sans cesse.

Empêchant tout fatalisme et tout irénisme, le principe de progrès et de régrès remet au centre la problématique de la volonté humaine, collective et individuelle. La révolution est déjà présente au sein de l'évolution comme l'indique Reclus lorsqu'il évoque le fait que « la révolution ne saurait tarder », puisqu'il ajoute : « D’ailleurs ne s'accomplit-elle pas constamment sous nos yeux, par multiples secousses? $? »^{128}$

\section{NOTES}

1. Alain Testart, «La question de l'évolutionnisme dans l'anthropologie sociale », Revue française de sociologie, XXXIII, 1992, p. 155-187.

2. Federico Ferreti, "Evolution and revolution: Anarchist geographies, modernity and poststructuralism", Environment and Planning D: Society and Space, $\mathrm{n}^{\circ}$ 35-3, 2017, p. 893-912.

3. Philippe Pelletier, Géographie \& Anarchie, Reclus, Kropotkine, Metchnikoff, Paris et Oléron, Éditions du Monde Libertaire et Éditions libertaires, 2013.

4. Plusieurs facteurs ont joué dans cette évolution somme toute assez rapide (1876-1880). L'idée de « communisme anarchiste » circule déjà chez certains communards réfugiés en Suisse, comme François Dumartheray (1876), ainsi que chez les internationalistes italiens (Cafiero, Malatesta, etc.). La prise de distance d'Andrea Costa (1851-1910), futur député socialiste (1882-1909), vis-àvis du courant anti-autoritaire (1877), croise la prise de distance de Carlo Cafiero vis-à-vis 
d'Engels, de Marx et de leurs partisans (1872), tout en entreprenant la rédaction d'un Abrégé du Capital (1879), salué par Marx.

5. Un premier livre est issu d'une conférence intitulée Évolution et révolution, et publiée en 1881 à Genève. Réédité en 2008 par Le Passager clandestin, avec une préface d'olivier Besancenot, il correspond à la sixième édition (Paris, La Révolte, 1891), disponible sur le site Gallica de la BNF ; infra É\&R. Il constitue la charpente d'un second livre qui le reprend sous une forme amplifiée et sous le nouveau titre de L'Évolution, la révolution et l'idéal anarchique, publié en 1897. Sa première édition se trouve sur le site de Cgecaf. Ses rééditions datent de 1898 (deux fois), 1902 (Paris, P.-V. Stock, Bibliothèque sociologique, $\mathrm{n}^{\circ}$ 19, 298 p., avec table des matières, version définitive), 1906, 1909, 1914, 1921 et 1931. Stock a réimprimé en 1979 l'édition de 1898. La sixième édition (Paris, Stock, 1906) est disponible sur le site Gallica. Reclus Élisée, L'Évolution, la révolution et l'idéal anarchique, Paris, Stock + Plus, 1979, 210 p., éd. or. 1898 (Paris, Stock, novembre 1897), septième édition revue et corrigée, sans table des matières, infra ÉRIA.

6. Élisée Reclus, L'Homme et la Terre, t. VI, chap. XII, « Progrès », Paris, Librairie Universelle, 1905, p. 501-541.

7. Lev Metchnikov, "Revolution and Evolution", Contemporary Review, 50, septembre, 1886, p. 412-437, infra É\&R. Metchnikoff Léon (1889) : "Le Progrès », La Civilisation et les grands fleuves historiques, Paris, Hachette, 372 p., chap. I, p. 6-28, infra CGFH.

8. ÉRIA, p. 7 et 9.

9. ÉRIA, p. 8.

10. Yvette Conry, L'Introduction du darwinisme en France au XIX $X^{e}$ siècle, Paris, Vrin, 1974, 480 p.

11. The Structure and distribution of coral reefs (1842) et Geological observations on South America (1846).

12. Paul Vidal de la Blache, La Terre, géographie physique et économique, histoire sommaire des découvertes, Paris, Delegrave, 1883, p. 103. Alfred de Lapparent, personnage renommé et professeur à l'Institut Catholique de Paris, s'oppose dès les années 1880 à la théorie darwinienne, et en 1885 traite spécifiquement de «la théorie des récifs coralliens». (Olivier Soubeyran, Imaginaire, science et discipline, Paris, L'Harmattan, 1997, 486 p., p. 340 et suiv.)

13. L'Homme et la Terre, t. II, p. 4, t. VI, p. 430-431.

14. Eugène Fournière, Les théories socialistes au XIX siècle, de Babeuf à Proudhon, Paris, Alcan, 1904, 416 p. Cité par Haubtmann, op. cit., p. 214.

15. Lettre du 19 décembre 1860.

16. Discours sur la tombe de Marx, prononcé par Engels en anglais à Highgate le 17 mars 1883. Publié en allemand dans le Sozialdemokrat, $\mathrm{n}^{\circ}$ 33, 22 mars 1883. Traduction en français publiée dans Karl Marx, Bureau d'Éditions, Paris, 1935.

17. Aux compagnons de l'Association internationale des travailleurs du Locle et de La Chaux-de-Fonds, Troisième Lettre du 14 avril 1869. Euvres, Paris, Stock + Plus,1980, vol. I, p. 251. Aux compagnons de la Fédération des sections internationales du Jura (février-mars 1872), Euvres complètes, vol. III, Paris, Champ libre, p. 57. Philippe Pelletier, «Bakounine géopolitique, esquisse ", Actualité de Bakounine, 1814-2014 (Ph. Pelletier coord.), Paris, Éditions du Monde libertaire, 2014, 178 p., p. 129-157.

18. L'Homme et la Terre, vol. VI, p. 418.

19. É\&R, p. 431.

20. Pierre Kropotkine, L'Éthique [1921], Paris, Stock, rééd. Tops-Trinquier, 2002, p. 38.

21. Federico Ferretti \& Philippe Pelletier, "Jules Vallès et Élisée Reclus, communalisme et anarchisme dans la révolution sociale ", Autour de Vallès, revue de lecteurs et d'études vallésiennes, $\mathrm{n}^{\circ}$ 46, 2016, p. 67-96.

22. Émile Gautier, Le Darwinisme social, Paris, Dervaux, 1880, 90 p.

23. Op. cit., p. 38, 39 et 40 .

24. Ibid., p. 77. 
25. En revenant à l'individu, base de départ et aboutissement du raisonnement sociologique et socialiste, Gautier affirme implicitement son positionnement anarchiste, qu'il n'évoque pas dans son livre. Il soutient l'idée de « collectiviser les moyens de production » et « d'individualiser les moyens de jouissance » (Ibid., p. 87, $\mathrm{n}^{\circ} 1$ ). Restant en quelque sorte dans le sillage du collectivisme bakouninien, il ne suit donc pas le «communisme anarchiste » proposé au même moment par Kropotkine, Cafiero et Reclus.

26. Linda L. Clark, Social darwinism in France, Tuscaloosa, University of Alabama Press, 1985, 262 p. Jean-Marc Bernardini, Le darwinisme social en France, fascination et rejet d'une idéologie, Paris, CNRS Éditions, 1997, $464 \mathrm{p}$.

27. Étant entendu que l'expression de social darwinism renvoyant généralement à la pensée de Herbert Spencer n'est pas utilisée par celui-ci, mais qu'elle lui a été appliquée plus tard, voire attribuée de façon anachronique.

28. Qu'il évoque, op. cit., p. 13.

29. Patrick Tort, La Pensée hiérarchique et l'évolution, Paris, Aubier, 1983, p. 51-58.

30. Bernard Naccache, Marx, critique de Darwin, Paris, Vrin, 1980, 160 p., p. 125.

31. Ernst Haeckel, Les Preuves du transformisme, réponse à Virchow, Paris, Germer Baillière, 1879, 166 p., p. 110 [Freie Wissenschaft und freie Lehre, 1877] ; Enrico Ferri \& R. R. La Monte, Socialism and modern science (Darwin, Spencer, Marx), Echo Library, 2006, 116 p., p. 7.

32. L'Homme et la Terre, t. VI, p. 430, chap. $\mathrm{x}$, « La religion et la science ».

33. L'Homme et la Terre, t. VI, p. 431.

34. Émile Gautier, op. cit., p. 2.

35. Lettre de juin 1888 d'Élisée Reclus à Renard, auteur d'un Essai sur le socialisme. Citée par Paul Reclus, Biographie d'Elisée Reclus [1939] rééditée in Les frères Élie et Élisée Reclus - ou du Protestantisme à l'Anarchisme, Paris, Les Amis d'Élisée Reclus, 1964, 212 p., p. 122.

36. Émile Gautier, op. cit., p. XIV.

37. Ibid., p. 28-29.

38. CGFH, p. 14.

39. Ibid., p. 17.

40. Ibid.

41. Pierre Kropotkine, Autour d'une vie, mémoires, Paris, Stock, 1898, 548 p., p. 515.

42. É\&R, p. 413.

43. Marianne Enckell, "Note sur l'histoire d'un mot", Réfractions, recherches et expressions anarchistes, $\mathrm{n}^{\circ} 23,2009$, p. 5-7.

44. É\&R, p. 26.

45. Ibid., p. 27.

46. Ibid., p. 28.

47. ÉRIA, p. 205.

48. CGFH., p. 8.

49. Georges Sorel traduit corsi et ricorsi par «suites" et "recommencements ». Georges Sorel, «Préface ». Histoire des Bourses du travail, origine, institutions, avenir [1901], Fernand Pelloutier, rééd. 1946, Paris, Alfred Costes, 342 p., p. 27-67, p. 34.

50. Pierre Haubtmann, Pierre-Joseph Proudhon, sa vie et sa pensée (1809-1849), Paris, Beauchesne, 1982, 1142 p., p. 249.

51. Gary Dunbar, Élisée Reclus, historian of Nature, Hamden, Archon Books, 1978, 200 p., p. 53.

52. L'Homme et la Terre, t. I, p. 344, 346, vol. VI, p. 526.

53. L'Homme et la Terre, t. V, vol. VI, p. 344.

54. L'Homme et la Terre, t. I, chap. vI, p. 346.

55. L'Homme et la Terre, t. V, vol. VI, chap. VI, p. 526.

56. Ibid.

57. L'Homme et la Terre, t. VI, p. 502. 
58. Les "peuples ", à mesure qu'ils se sont "développés en intelligence et en liberté », sont «devenus, par la force de l'association, de véritables agents géologiques [qui] ont transformé de diverses manières la surface des continents, changé l'économie des eaux courantes, modifié les climats eux-mêmes » (« Du sentiment de la nature dans les sociétés modernes », La Revue des Deux Mondes, t. LXIII, 15 mai 1866.)

59. L'Homme et la Terre, t. VI, fin du chap. III, p. 164.

60. L'Homme et la Terre, t. VI, p. 515.

61. L'Homme et la Terre, t. VI, p. 510.

62. Ce livre d'Engels et son inspiration ont été passés au tamis critique d'un économiste anthropologue marxiste qui, au vu des travaux récents, aboutit logiquement à leur sévère remise en cause sur le fond, quoi qu'il s'en prévienne. Il fait cependant quasi totalement l'impasse sur les travaux d'anthropologues proches de l'anarchisme comme Alfred Radcliffe-Brown (1881-1955) (anarchiste dans sa jeunesse), Harold B. Barclay (1924-2017), Pierre Clastres (1934-1977) ou les contemporains David Graeber (né en 1961) et James C. Scott (né en 1936). Christophe Darmangeat, Le communisme primitif n'est plus ce qu'il était, aux origines de l'oppression des femmes, Toulouse, Smolny, 2009, 466 p.

63. Alaisn Testart, op. cit., p. 170.

64. L'Homme et la Terre, t. VI, dernier chapitre « Le Progrès ».

65. Élie Reclus, «Primitifs », 1885. Élisée Reclus : «peuples attardés », L'Homme et la Terre, I, 165 ; «tribus sauvages ", L'Homme et la Terre, I, 14 ; «monde encore réputé sauvage »: L'Homme et la Terre, VI, 514 ; « races dites “inférieures" »: L'Homme et la Terre, VI, 366 ; « nos frères primitifs » : L'Homme et la Terre, VI, 510 ; « sociétés dites sauvages ou barbares » : L'Homme et la Terre, VI, 511. Kropotkine : «Les Sauvages », « peuples primitifs », « nos sauvages contemporains » (1902); «le sauvage primitif », « l'homme primitif », " peuples sauvages ", « société primitive » (1921).

66. Par exemple, en ce qui concerne Vidal, dans Principes de géographie humaine, 1921, rééd. de 1995, p. 34.

67. Philippe Pelletier, « Géographie, anthropologie et anarchie au XIX ${ }^{\mathrm{e}}$ siècle, carrefours, rendezvous manqués et promesses ", Journal des Anthropologues, $n^{\circ} 152-153,2018$, p. 35-56.

68. La Civilisation et les grands fleuves historiques (infra, désormais, CGFH) (1889), p. 98.

69. L'Entr'aide, un facteur de l'évolution (infra, désormais, EFÉ), éd. angl. (1902), éd. or. fr. (1906), rééd., Paris, Éditions de l'Entr'aide, 368 p., p. 91.

70. L'Homme et la Terre, V, p. 329 et sv.

71. EFÉ, p. 105.

72. Ibid., p. 90.

73. Nouvelle Géographie Universelle (infra, désormais, NGU), XII, L'Afrique Occidentale, Archipels atlantiques, Sénégambie et Soudan Occidental, Paris, Hachette, 1887, p. 264.

74. Ibid., p. 469.

75. NGU, XIII, L'Afrique Méridionale (1888), p. 199.

76. EFÉ, p. 121.

77. L'Homme et la Terre, VI, p. 512.

78. L'Éthique, op. cit., p. 71.

79. Philippe Pelletier, Le Japon, une merveille de l'histoire vu par Élisée Reclus et Léon Metchnikoff, Tôkyô, Ebisu, 2019, 62 p.

80. L'Homme et la Terre, V, p. 428.

81. L'Homme et la Terre, V, p. 270.

82. «Considérations sur quelques faits de géologie et d'ethnographie: Histoire du sol de l'Europe, par M. Houzeau ", Revue philosophique et religieuse, Paris, $1^{\mathrm{er}}$ janvier 1858, p. 218-227. Rééd. in Christophe Brun Élisée Reclus, les grands textes, Paris, Flammarion, 2014, p. 204.

83. «Une conférence d'Élisée Reclus sur l'Angleterre, la Russie, l'Afghanistan », La Justice, 9 juin 1885 , p. 2, d'abord publié par Le Petit Colon d'Alger. La Justice est un quotidien parisien fondé en 
1880 par des républicains radicaux (Clemenceau, Pelletan) hostiles à l'expansion coloniale de Jules Ferry.

84. L'Homme et la Terre, VI, p. 208.

85. L'Homme et la Terre, VI, p. 515.

86. L'Homme et la Terre, VI, p. 5.

87. Élie Reclus, Les Primitifs, études d'ethnologie comparée, Hyperboréens orientaux et occidentaux. Apaches monticoles des Nilgherris, Naïrs, Khonds, Paris, Chamerot, 1885, p. XIII.

88. L'Homme et la Terre, VI, p. 531.

89. Ibid., p. 538.

90. L'Homme et la Terre, I, p. 122.

91. Ibid.

92. « Pages de sociologie préhistorique », 1898, et L'Homme et la Terre, I, p. 126.

93. L'Homme et la Terre, t. VI, p. 502. Gibbon est connu pour The History of the Decline and Fall of the Roman Empire. 6 vol. (1776-1788).

94. Ibid., p. 506.

95. Ibid., p. 504.

96. Paul Mougeolle, Les Problèmes de l'histoire, relations des faits dans l'espace et dans le temps, les hommes, les sociétés, les races, les dieux, Paris, C. Reinwald, 1886, 520 p., cité par Léon Metchnikoff, op. cit., p. 8-9.

97. L'Homme et la Terre, t. VI, p. 504.

98. Ibid., p. 503.

99. Ibid.

100. Ibid.

101. L'Homme et la Terre, t. VI, p. 508.

102. L'Homme et la Terre, t. VI, p. 506.

103. Jean-Marie Guyau, Morale d'Épicure, 1879, p. 153 et suiv., L'Homme et la Terre, t. VI, p. 506.

104. Ainsi ce propos de Jean-Marie Pelt : «Si nous sommes lucides, nous ne pouvons que voir que nous sommes actuellement dans le mythe de Prométhée, dans une phase de l'Histoire où le progrès scientifique repousse les limites de la puissance de l'homme et où l'homme, se posant luimême en demi-dieu, abandonne Dieu. C'est particulièrement visible dans le mouvement contemporain des sciences, de la technologie et de l'économie: l'homme y a pris la place de Dieu ». Alliance pour une Europe, 16, janvier-février 2008.

105. Jean-Pierre Lecercle, «La notion de littérature anarchiste ", Conférences en français de la RIA, 8-12 août 2012, Paris, Place d'armes, 2013,132 p., p. 47-130.

106. Ibid.., p. 96.

107. Élisée Reclus, « Aux compagnons rédacteurs des Entretiens », Entretiens politiques et littéraires. L'Homme et la Terre, t. VI, 1892, p. 507.

108. Première phrase introduisant « Du sentiment de la nature dans les sociétés modernes ", $L a$ Revue des Deux Mondes, t. LXIII, 15 mai 1866, p. 351-357 et 371-377.

109. L'Homme et la Terre, t. VI, p. 508.

110. Ibid.

111. Ibid., p. 523

112. L'Homme et la Terre, t. VI, p. 531.

113. Ibid., p. 525-526

114. Ibid., p. 527.

115. É\&R, p. 53.

116. ÉRIA, p. 97-98.

117. L'Homme et la Terre, t. VI, p. 528.

118. L'Homme et la Terre, t. VI, p. 534.

119. L'Homme et la Terre, t. VI, p. 530. 
120. L'Homme et la Terre, t. VI, p. 540-541.

121. Nicholas Spencer, "Historicizing the spontaneous revolution: anarchism and the spatial politics of postmodernism», 1997, < http://www.ags.uci.edu/ clcwegsa/revolutions/ Spencer.htm >.

122. Juan Salvador, Critique de la déraison évolutionniste, animalisation de l'homme et processus de civilisation, Paris, L'Harmattan, 2006.

123. Philippe Pelletier, La Critique du productivisme dans les années 1930, mythe et réalités, Paris, Noir \& Rouge, 2015, 186 p.

124. "Préface", avec Carlo Cafiero, de Dieu et l'État de Bakounine, Genève, Imprimerie jurassienne, 1882.

125. ÉRIA, chap. VIII.

126. Faute d'une véritable connaissance de la pensée reclusienne et de l'anarchisme, Salvador Juan les analyse malheureusement de façon grossière dans son livre. Ses approximations relèvent de cette lecture " post-anarchiste » erronée que décortique Federico Ferretti, op. cit. En revanche, Juan vise juste en pointant l'animalisation actuelle de l'humain et du social, ce qu'esquissaient déjà Reclus ou Kropotkine dans leur critique du social-darwinisme.

127. Alaisn Testart, op. cit., p. 164.

128. ÉRIA, op. cit., p. 205.

\section{RÉSUMÉS}

Pour Élisée Reclus (1830-1905), géographe anarchiste, il n'y a pas de philosophie de l'histoire mais des variations de milieux (physiques, humains) faits de progrès et de régrès. Le progrès est une amélioration de la condition humaine: il «consiste à trouver l'ensemble des intérêts et des volontés commun à tous les peuples, il se confond avec la solidarité ». Nulle situation n'est figée puisque chaque milieu étant localement divers, chaque peuple peut effectuer des choix techniques et culturels différents malgré des conditions parfois proches. Reclus critique aussi bien les contempteurs que les laudateurs du progrès.

For Élisée Reclus (1830-1905), the philosophy of history does not exist but there are many variations of « milieux » (physical and human) constitued by progress and « regress ». Progress is a way for improvement of human condition: it "means find the whole of interest and will common to all peoples, merged with solidarity ». No situation is fixed since each milieu is locally diverse, each people making technical and cultural choices in spite of similar conditions. Reclus is critical both towards people who admire or reject progress.

\section{INDEX}

Mots-clés : progrès, régrès, civilisation, milieux

Keywords : progress, regress, civilization, milieu 
AUTEUR

PHILIPPE PELLETIER

Université Lyon 2, UMR 5600 Environnement, ville, société 